\title{
Automated Topology Optimization of Hybrid Electric Vehicle Powertrains
}

\begin{abstract}
:
Hybrid Electric powertrains are comprised of gasoline and electric powertrains components. These components can be connected in numerous configurations; as the number of components increase, the number of configurations increase exponentially. In this research, an algorithm was developed to automatically assemble and compare all possible hybrid electric powertrain configurations. Combinatorics was used to discover all possible combinations of: an internal combustion engine, high-torque low-speed electric motor, low-torque high-speed electric motor, planetary gearset, five-speed discrete gearbox, and battery. The Graph Theoretic Method was used to generate the powertrain models. The steadystate system models were solved symbolically, and Dynamic Programming was used to determine the optimal control law that minimized fuel consumption. To save computation time, topologies were evaluated using a multi-stage screening process. The component sizes of the top 24 topologies were optimized and ranked by fuel consumption. It was found that the Parallel and Powersplit-like topologies with discrete gearboxes were most efficient. The best performing topology is a Powersplit Hybrid type, with a discrete gearbox connected to the final drive and the output gear of the planetary carrier and electric motor in parallel.
\end{abstract}

Keywords: hybrid electric vehicles; hybrid electric powertrains; topology optimization; linear graph theory; Graph Theoretic Method; design automation

\section{Biographical notes:}

\section{Introduction}

Conventional transportation vehicles use combustion engines for propulsion because fossil fuels are energy dense and relatively inexpensive. Rising fuel prices, depletion of fossil fuels, and public environmental awareness have made electric vehicles an attractive alternative. Electric vehicles use electricity instead of gas for propulsion. They are more efficient, quieter, and do not create tailpipe emissions. Unfortunately, conventional electric vehicles are heavily reliant on batteries for energy storage. Batteries are expensive, heavy, and require charging equipment. Fuel cells have been proposed as an alternative to batteries, but hydrogen fuelling infrastructure is not widely available, and high pressure hydrogen storage technology is not mature. As an intermittent step from gasoline to electric vehicles, Hybrid Electric Vehicles (HEVs) have emerged in the marketplace.

Hybrid Electric powertrains combine elements from traditional gasoline powertrains and electric powertrains. This is advantageous because they operate 10-20\% more efficiently, and can refuel at any gasoline station (Chen et al., 2009; del Re et al., 2010; Chan, 2007). Including additional powertrain components create challenges. Complex controllers are required to ensure the system is operating efficiently. Cost and weight also increase with the number of components.

The number of potential powertrain configurations increase exponentially as more components are included. Novel powertrain architectures can be identified through human 
judgement or numerical enumeration. Human judgement relies on experience, and is subject to human error and cognitive bias. Enumerative methods involve numerically generating then testing all possible permutations, which can require impractically large amounts of computing power. Evaluating topologies involve building prototypes or performing modelling and simulation. Mathematical models are faster and cheaper to create and allow us to reduce the number of prototypes built by identifying optimal starting points. This paper presents a framework to automate the design of a HEV powertrain. Due to practical time constraints, the design space is limited to HEV powertrains comprised of: an internal combustion engine (ICE), high-torque low-speed electric motor (EM), low-torque highspeed electric motor (GEN), planetary gearset/device (PSD), five-speed discrete gearbox (GB), and battery (BAT). The main techniques used in this approach are: the Graph Theoretic Method, Dynamic Programming, and Pattern Search Optimization.

\subsection{Hybrid Electric Vehicle Architectures}

The most common HEV architectures are Parallel, Series, and Powersplit hybrids. Parallel Hybrids are the simplest configuration because they are architecturally similar to a traditional gasoline vehicle. The engine is connected through a gearbox to the wheels, and is in parallel (shares a driveshaft) with an electric motor. The main advantage of this architecture is its simplicity; there are few added components making this a cheap, light alternative. Compared to other hybrids, this configuration may be less efficient because the engine is mechanically connected to the wheels; the optimal engine operating speed may not always be chosen (Ehsani et al., 2010). The Honda Civic Hybrid and Honda Insight are examples of Parallel hybrids (Chan, 2007).

Series Hybrids are also known as range extended EVs. The Series Hybrid effectively operates as an EV by powering the electric motor with the battery. When the battery's StateOf-Charge is low, the gasoline engine will start and run the generator to generate electricity used to charge the battery. The Series architecture allows the engine to operate at the most efficient speed at all times, but introduces losses associated with converting mechanical power to electrical power, and back to mechanical power (Ehsani et al., 2010). Most electric buses operate using the Series hybrid architecture.

Powersplit Hybrids are also known as Series-Parallel hybrids because they can operate both as a Series hybrid or a Parallel hybrid. The most common passenger hybrid, the Toyota Prius, is build upon this architecture.

The Powersplit powertrain has a planetary gearset that allows for multiple operating modes. For example, a Prius-like Powersplit powertrain will operate as a Parallel hybrid if the sun gear is locked; the planetary gearset reduces to a simple gear ratio, and the engine will provide traction through the ring gear to the final drive. Alternatively, when the sun gear is not locked, the engine will turn the generator to generate electricity like a Series Hybrid (Ehsani et al., 2010; Bayrak et al., 2013; Kawahashi and Toyota Motor Corporation, 2004). This flexibility makes the Powersplit Hybrid very efficient and thus the basic architecture has been adopted (with some modifications), for commercial application by Toyota, General Motors, and Ford.

The Ford Hybrid System, Toyota Hybrid System I \& II, and GM-Allsion Hybrid system use variations of the Powersplit hybrid system, but include clutches and/or multiple planetary gearsets (Bayrak et al., 2013; Kawahashi and Toyota Motor Corporation, 2004; Miller and Everett, 2005). The focus on this paper is only on full hybrids-hybrids that can be driven on all electric power. 


\subsection{Literature Review}

Heuristics or human judgment can be applied to assess the feasibility of a topology (Bayrak et al., 2013). If a powertrain architecture is feasible, it can be further improved by optimizing the size of its components. This section will describe previous methods used by other authors to generate and evaluate $\mathrm{HEV}$ powertrain topologies.

Bayrak et al. (Bayrak et al., 2013) described a systematic method to select the optimal configuration for an architecture containing two planetary gearsets, one engine and two electric motors. The topology is represented using bond graphs, and the system equations are generated in state space form. Using this method, an architecture was found that improves the fuel consumption by $8 \%$ compared to a Prius-like configuration.

Liu et al. (Liu and Peng, 2010) proposed an exhaustive search approach to HEV configurations involving two planetary gears, an ICE, and two electric motors. The powertrain is designed for a military vehicle (Humvee), with a curb weight of approximately $5000 \mathrm{~kg}$. Each configuration is evaluated for structural feasibility, component size is optimized (including gear ratio, but not battery), and drive performance is evaluated. Configurations are evaluated by fuel consumption using Dynamic Programming.

Ma et al. (Ma et al., 2013) performed a kinematic analysis of HEV powertrains including two planetary gear sets and two clutches. All permutations are evaluated for top speed and torque. It was found that the second planetary gearset can act as a variable reduction gear; thus better accelerations can be achieved at higher velocities.

Zhang et al. (Zhang et al., 2012) used an enumerative method to compare powersplit hybrid powertrains for Prius-like and Volt-like HEV powertrains. The configurations contained: one planetary gear, an ICE, two electric motors, and any number of clutches. Removing a clutch from the Volt configuration (originally 3 clutches) resulted in similar fuel consumption for two different drivecycles, and thus a clutch can theoretically be removed to simplify the design of the Volt powertrain.

Hofman et al. (Hofman et al., 2012) used a manual search method to optimize the location and size of the transmission, electric motor and ICE. Viability of the topology was manually assessed, then equations were manually assembled. Dynamic Programming is used to find the optimal control law that minimizes $\mathrm{CO}_{2}$ emissions over a prescribed drive cycle. It was found that the optimal topologies were an automatic transmission with electric motor between ICE/transmission, or a continuously-variable transmission with an electric motor between transmission/differential.

\subsection{Research Contributions}

Of the reviewed work, Linear Graphs and the Graph Theoretic Method have yet to be applied to HEV topology optimization. Novel heuristics used to screen potential topologies are also presented in this paper. When used together, an automated approach to synthesize and optimize Hybrid Electric Vehicle powertrains is achieved. Liu (Liu, 2004) first proposed similar work focusing on the automated synthesis of planar mechanisms, named this computer implementation 'AutoSyn'. As tribute, we have named this computer implementation ASHev - AutoSyn for Hybrid Electric Vehicles. 


\section{Hybrid Electric Powertrain Component Models}

The HEV powertrain components are described in this section. Longitudinal vehicle dynamics, the final drive reduction gear losses, internal combustion engine, electric motor/generator, Powersplit device and battery are described using steady-state models; thus all the equations are algebraic. Where possible, data was collected from the Autonomie 2012 software package (Argonne National Labs, 2012) for use in the ASHev model. It is assumed that the vehicle can reach any operation point within 1 second. Scoring penalties to prevent unrealistic behavior are introduced in Dynamic Programming. At the end of the section, the scaling equations for the components are described.

\subsection{Vehicle Dynamics}

The vehicle is described by its longitudinal dynamics (del Re et al., 2010; Lino Guzzella, 2013; Wang and Lukic, 2012; Rodrigo Garvia-Valle, 2013; Irani, 2009). This assumes the vehicle is driving forward in a straight line on a flat plane on a known slope. Lateral effects such as turning and crosswinds are ignored. The longitudinal dynamics are described in equation 1 , where $m_{v}$ is the vehicle mass (including powertrain), $v(t)$ is the vehicle velocity, $F_{a}$ is aerodynamic drag, $F_{r}$ is the rolling friction, $F_{g}$ is component of weight along the sloped roads, and $F_{d}$ is the inertial force of the driveline. $F_{t}$ is the traction force generated by the motor or generator, which is transmitted through the final drive to the wheels.

$$
F_{t}(t)=m_{v} \frac{d}{d t} v(t)+F_{a}(t)+F_{r}(t)+F_{g}(t)+F_{d}(t)
$$

Aerodynamic forces are described in equation 2 , where $\rho$ is the density of air, $A_{f}$ is the frontal area of the vehicle, and $c_{d}$ is the coefficient of drag, which is assumed to be constant.

$$
F_{a}(v)=\frac{1}{2} \rho A_{f} c_{d} v^{2}
$$

Rolling friction is described by equation 3, where $c_{r}$ is the coefficient of rolling friction (assumed to be constant), $g$ is the acceleration due to gravity, and $\alpha$ is the slope of the road.

$$
F_{r}=c_{r} m_{v} g \cos (\alpha)
$$

The force of gravity is described equation 4 .

$$
F_{g}=M g \sin (\alpha)
$$

The inertial driveline force is described by equation 5 , where $\lambda$ is the rolling inertia of the driveline (assumed to be a constant 1.089 [-] (del Re et al., 2010)).

$$
F_{d}=\lambda m_{v} \frac{d v}{d t}
$$




\subsection{Final Drive}

The final drive (FD) model is a simple reduction gear assuming a constant $3 \%$ power loss (Argonne National Labs, 2012). The equation for torque and speed are seen in equation 6.

$$
\begin{aligned}
\tau_{F D} & =\frac{\tau_{\text {Wheel }}}{0.97 i_{0}} \\
\omega_{F D} & =\omega_{\text {Wheel }} i_{0}
\end{aligned}
$$

where $\tau_{F D}$ and $\omega_{F D}$ is the final drive torque and angular speed; $\tau_{W h e e l}$ and $\omega_{W h e e l}$ is the torque and angular speed of the wheel; $R_{W h e e l}$ is the wheel radius; and $i_{0}$ is the ratio of the reduction gear for the final drive.

\subsection{Internal Combustion Engine}

The Internal Combustion Engine (ICE) burns gasoline in a small chamber containing a piston connected to a crankshaft. The expansion of gas causes the piston to move, thus forcing the crankshaft to rotate. The engine efficiency is generally determined experimentally and described using a Brake Specific Fuel Consumption (BSFC) map (shown in figure 1). In this paper, the ICE fuel consumption is calculated by interpolating the experimentally validated BSFC map taken from Autonomie (Argonne National Labs, 2012). This steady-state model assumes the engine is always hot. The fuel consumption rate is a function of the ICE torque and speed and can be described by the equation:

$$
\dot{m}_{f u e l}=B S F C\left(\tau_{I C E}, \omega_{I C E}\right) \tau_{I C E} \omega_{I C E}
$$

The maximum torque constraint is described using a 3rd order polynomial in the form (Wang and Lukic, 2012; del Re et al., 2010):

$$
\tau_{I C E \max }=p_{1} x^{3}+p_{2} x^{2}+p_{3} x+p_{4}
$$

\subsection{Electric Motor / Generator}

Usually 3-3 polynomials are sufficient to estimate the efficiency of an Electric Motor/Generator (EM/GEN) (Mcdonald, 2013). However, it was found that a 4-4 polynomial had a better fit to the efficiency maps (Argonne National Labs, 2012). The EM/GEN electrical power requirement is modelled as a 4-4 polynomial described in equation 9.

$$
\begin{gathered}
\eta_{E M}=p_{00}+p_{10} \tau+p_{01} \omega+p_{20} \tau^{2}+p_{11} \tau \omega+p_{02} \omega^{2}+p_{30} \tau^{3}+p_{21} \tau^{2} y \\
+p_{12} \tau \omega^{2}+p_{03} \omega^{3}+p_{40} \tau^{4}+p_{31} \tau^{3} \omega+p_{22} \tau^{2} \omega^{2}+p_{13} \tau \omega^{3}+p_{04} \omega^{4}
\end{gathered}
$$

where $\tau$ and $\omega$ are the torque and speed of the EM/GEN respectively. The resultant fit of $R^{2}$ $=0.9995$ is acceptable, but will accumulate errors over time. The EM/GEN acts as both a motor and generator, each mode a with different efficiency. If the product of the torque and speed is positive (first and third quadrant), it is acting as a motor. If the product is negative (second and fourth quadrant), then it is acting as a generator. This model behaviour is shown 


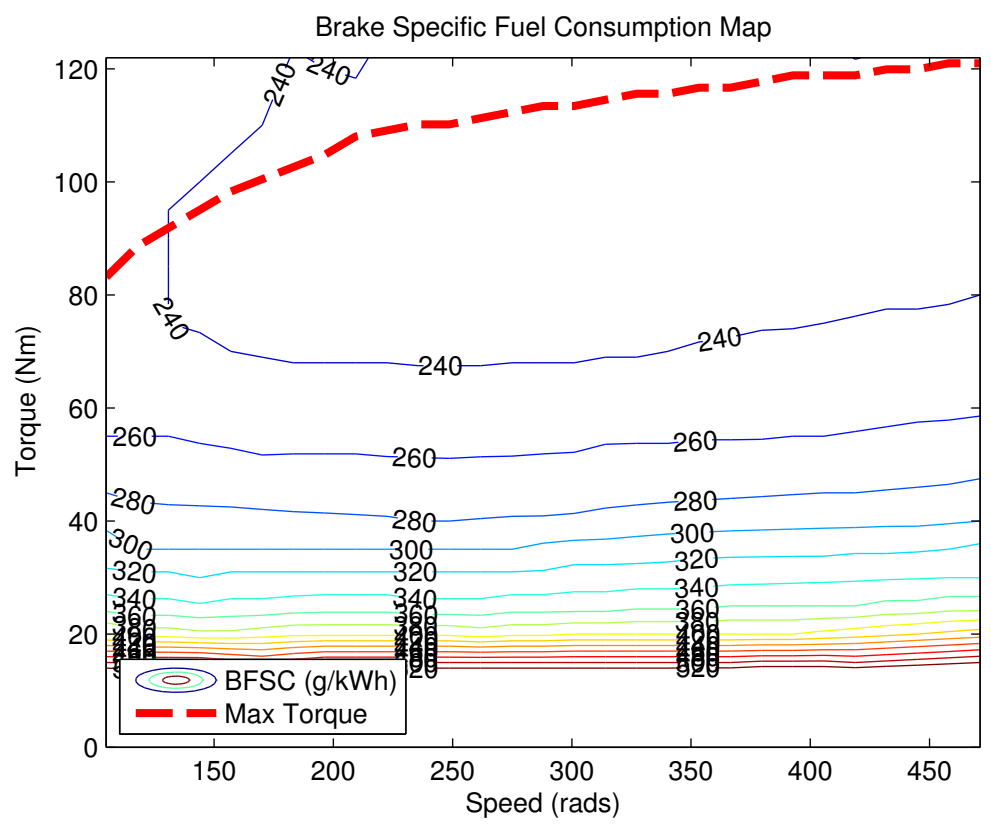

Figure 1 Brake Specific Fuel Consumption Hot Map for 57kW internal combustion engine (Argonne National Labs, 2012).

in figure 2. Shown in equation 10 , a piecewise equation is required to the behaviour under different conditions.

$$
P_{E M}(\tau, \omega)=\left\{\begin{array}{l}
0, \tau \omega=0 \\
+\eta_{E M}(\tau, \omega) \tau \omega, \tau \omega>0 \\
-\eta_{E M}(\tau, \omega) \tau \omega, \tau \omega<0
\end{array}\right.
$$

where $+\eta_{E M}$ and $-\eta_{E M}$ are the efficiency of the motor working as a motor $(+)$ and generator $(-)$ respectively.

\subsection{Discrete Gearbox}

The 5-speed manual gearbox (GB) is comprised of a train of spur gears (Harald Naunheimer, Bernd Bertsche, Joachim Ryborz, 1994). Such gears are commonly used in automotive applications because they are 98-99\% efficient (Norton, 1992). Depending on the selected gear, the gearbox can act as either a speed reducer or a speed multiplier. The 5-speed gearbox is modelled as an lossless gear (Argonne National Labs, 2012) because the system equations are acausal and the gearbox will not know which flange is the input/output. The gearbox equations are shown in equation 11.

$$
\begin{aligned}
\tau_{G B b} & =\frac{-1}{R_{G B}\left(i_{g}\right)} \tau_{G B a} \\
\omega_{G B b} & =R_{G B}(i g) \omega_{G B a}
\end{aligned}
$$

where $G B a$, and $G B b$ are rotational flanges on the gearbox, $R_{G B}$ is the gear ratio equal to one of $\{3.32,2,1.36,1.01,0.82\}$, and $i_{g}$ is the gear number. 


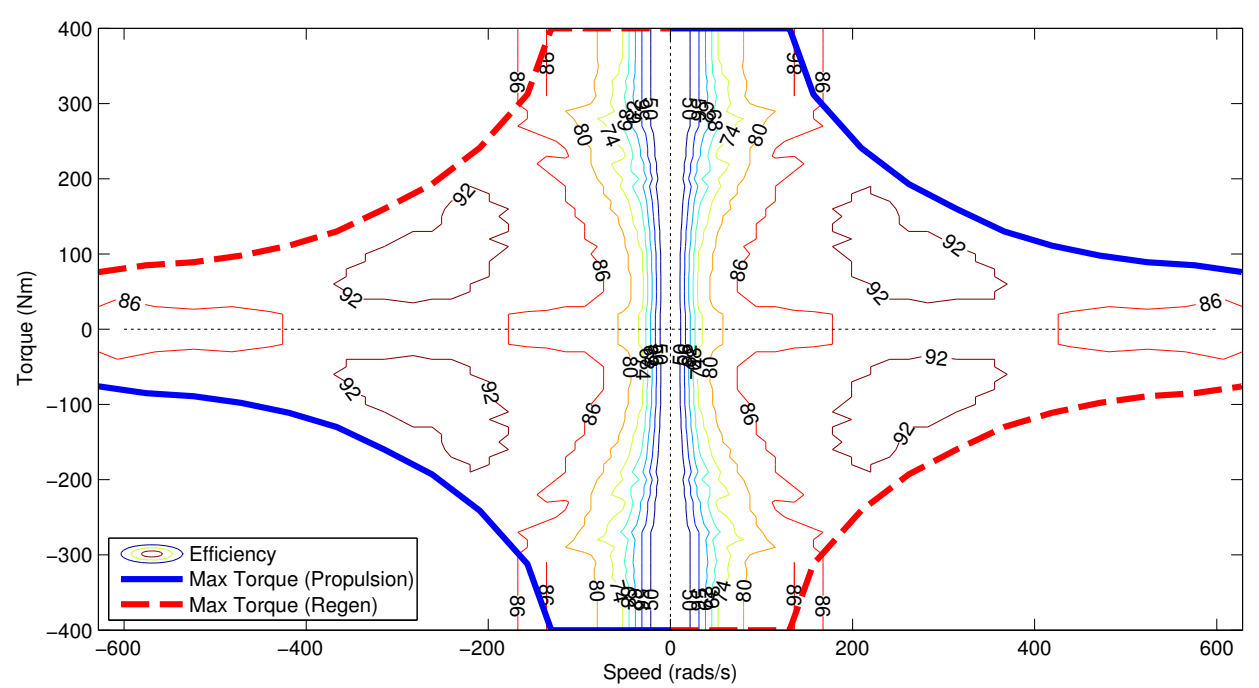

Figure 2 Electric motor efficiency map.

\subsection{Powersplit Device}

The Powersplit Device (PSD), also known as the planetary gearset, can be found in: automatic gearboxes, automotive differentials, and aircraft propeller reductions. The PSD allows 3 rotational components to be connected together. The Powersplit Device is comprised of a ring gear, a sun gear, a planetary carrier gear, and the planet gears. As the sun gear turns, it will force the planet gears to turn in the opposite direction, which turns the ring gear. The planet carrier can also turn, which causes the planet gears to rotate around the sun gear allowing for different speed outputs (including negative speeds) at the ring gear (Norton, 1992; Harald Naunheimer, Bernd Bertsche, Joachim Ryborz, 1994).

The Powersplit Device is described using 6 variables and 3 algebraic equations, thus allows 3 Degrees-of-Freedom (DOF). If one of the gears is locked the system become a single speed gear. In the case of a Powersplit hybrid, the speed of one of the rotational flanges is specified as a function of vehicle (wheel) speed, so this system is reduced to a 2 DOF system. The PSD is modelled as a lossless device, shown in equation 12, where $R, S$, $P C$ represent the ring, sun, and planetary carrier gear, respectively; $R_{P S G}$ is the ratio of teeth on the sun gear to the ring gear where, $R_{P S G}<1$.

$$
\begin{gathered}
\tau_{P S D_{R}}-R_{P S G} \tau_{P S D_{S}}=0 \\
\tau_{P S D_{P C}}+\tau_{P S D_{S}}+\tau_{P S D_{R}}=0 \\
\omega_{P S D_{P C}}\left(1+R_{P S G}\right)-\omega_{P S D_{S}}-R_{P S G} \omega_{P S G_{R}}=0
\end{gathered}
$$

\subsection{Battery}

The battery is generally considered the most difficult component in a hybrid electric powertrain to model due to its non-linear electrochemical nature. The battery terminal voltage is affected by battery temperature, age, and State-Of-Charge (SOC) (Gomez et al., 2011; Johnson, 2002). Shown in in equation 13, the Equivalent Circuit Battery Model is one of the simplest battery models (Argonne National Labs, 2012; Johnson, 2002). The open 
circuit voltage $\left(V_{o c}\right)$ and internal resistance $\left(R_{i n t}\right)$ are modelled as static functions of the battery State-Of-Charge (SOC) based on empirical data. As the terminal voltage is only a function of the SOC, the voltage drops will not be reported during high output transients.

$$
\begin{aligned}
& V_{o c}, R_{\mathrm{int}}=f(S O C) \\
& V=\frac{V_{o c}+\sqrt{V_{o c}{ }^{2}-4 R_{\mathrm{int}} \eta_{\text {inv }} P_{\text {batt }}}}{2} \\
& S O C_{k+1}=S O C_{k}-\frac{P}{3600 V Q_{\text {battcap }}}
\end{aligned}
$$

where $\eta_{i n v}$ is the inverter efficiency (assumed to be a constant of 0.95), $P_{b a t t}$ is the battery power, $S O C$ is the State-of-Charge of the battery, and $Q_{b a t t c a p}$ is the maximum battery capacity in Ampere-hours.

\subsection{Component Masses and Scaling}

A common way to represent a different sized component is to linearly scale the model. Most of the components described in this chapter can be scaled for performance and mass. For example, the ICE could be scaled up by 1.5 times by multiplying: the BFSC map by 1.5 , the maximum torque constraint by 1.5 , and the ICE mass by 1.5 . The baseline masses and scaling factors for each component is shown in table 1 . The $S$ symbol denotes how the scaling factor is applied to the original data. The planetary gear was not scaled, and the discrete gearbox can scale the gear ratios while maintaining a constant mass.

The ICE, EM and GEN use baseline performance maps for a 2004 Prius. The first equation linearly scales the power requirement, i.e. $B S F C$ (gasoline) for the ICE, and $P_{E M}$ and $P_{G E N}$ (electrical power) for the electric motor and generator. For the electric motor, the appropriate maps are scaled before curve-fit to polynomials. The second equation describes their maximum output constraint (torque).

As the number of battery cells is assumed to be constant, the capacity of these cells will scale with the scaling ratio, but the nominal voltage is kept constant.

In this section the equations for a vehicle and its components were described. The components can be scaled linearly to represent different sized components. Further details can be found in the thesis (Ing, 2014). When assembled correctly, these equations can be used modularly and be made to represent a number of different HEV powertrain architectures. The next chapter will explain how the architectures are represented and the vehicle equations generated.

\section{System Model and Validation}

The vehicle system topology is assembled using Linear Graph Theory, and the Graph Theoretic Method is used to automatically generate system models. It is assumed the reader is familiar with these methods, and is referred to (McPhee, 1996, 2005, 2004) for a more detailed explanation. At the end of this section, a model of a Parallel Hybrid is created and validated against a model provided by Autonomie. 
Table 1 Baseline Mass and Scaling Equations for HEV Components.

\begin{tabular}{|c|c|c|c|}
\hline Component & $\begin{array}{l}\text { Baseline mass } \\
(\mathrm{kg})\end{array}$ & Baseline & Scaling Equations \\
\hline ICE & $34.00 * \mathrm{~S}$ & $\begin{array}{l}\text { Prius MY04 } \\
57 \mathrm{~kW} \\
1.5 \mathrm{~L} 4 \text { Cylinder }\end{array}$ & $\begin{array}{l}B S F C=B S F C * S \\
\tau_{I C E_{\max }}=\tau_{I C E_{\text {max } \text { base }}} * S\end{array}$ \\
\hline$\overline{\mathrm{EM}}$ & $86.76 * \mathrm{~S}$ & $\begin{array}{l}\text { Permanent Magnet } \\
50 \mathrm{~kW} \text { peak } \\
25 \mathrm{~kW} \text { continuous }\end{array}$ & $\begin{array}{l}P_{E M}=P_{E M} * S \\
\tau_{E M_{\max }}=\tau_{E M_{\max , \text { base }}} * S\end{array}$ \\
\hline GEN & $25.00 * \mathrm{~S}$ & $\begin{array}{l}\text { Permanent Magnet } \\
30 \mathrm{~kW} \text { peak } \\
14 \mathrm{~kW} \text { continuous }\end{array}$ & $\begin{array}{l}P_{G E N}=P_{E M} * S \\
\tau_{G E N_{\max }}=\tau_{G E N_{\text {max }, \text { base }}} * S\end{array}$ \\
\hline GB & 75.00 & {$[3.32,2,1.36,1.01,0.82]$} & $R_{G B}=R_{G B, \text { base }} * S$ \\
\hline BAT & $35.70 * \mathrm{~S}$ & $\begin{array}{l}\text { NiMH } \\
\text { 6.5Ah 51xSeries@273V }\end{array}$ & $B A T_{C a p}=B A T_{C a p, b a s e} * S$ \\
\hline PSD & 40.00 & Ring/Sun teeth ratio $=78 / 30$ & - \\
\hline
\end{tabular}

\subsection{Topology Representation and Equation Formulation}

The topology of a vehicle is represented by an incidence matrix (IM) consisting of only zeros and ones; ones represent a connection, and zeros represent no connection. The matrix can take many possible permutations ; however, only a limited number of incidence matrices result in valid systems of equations. The information in a incidence matrix is represented using a 'genome'. A genome is the IM written as a linear string form and contains information describing both the mechanical and electrical topology.

The IM is generated by formatting the genome into matrices. The IM dimensions are dependent on the number of components (columns), and the number of nodes (rows). The first part of the genome represents the mechanical graph, and the second part the electrical graph. Component sizes are handled in a separate vector. In the example shown in equation ??, the mechanical incidence matrix is set to be a 3x7 matrix, and the electrical incidence matrix is set to be $1 \times 3$. From the IMs, the equations can be automatically generated (McPhee, 1996, 2005, 2004).

\subsection{Validation for Parallel Hybrid Powertrain Architecture}

Numerous architectures were validated, but only results for the Parallel architecture is presented for brevity. The Parallel architecture was validated by: (i) creating the desired architecture to be validated in Autonomie, (ii) running the forward-dynamic simulation in Autonomie, (iii) extracting the inputs and outputs from Autonomie, (iv) using the inputs from Autonomie as inputs in ASHev, and (v) comparing the outputs between Autonomie and ASHev. If the outputs of the two simulations are similar, then the model is valid. Differences in the model features and assumptions are highlighted below in table 2. It should be noted that both models use the same maps for electric motor/generator power, and ICE fuel consumption. They are linearly scaled to fit the required component size. The Parallel hybrid powertrain architecture was validated using the parameters in table 3 . The simulation outputs are shown in figure 4 . The final drive torque and speed were specified so their graphs 


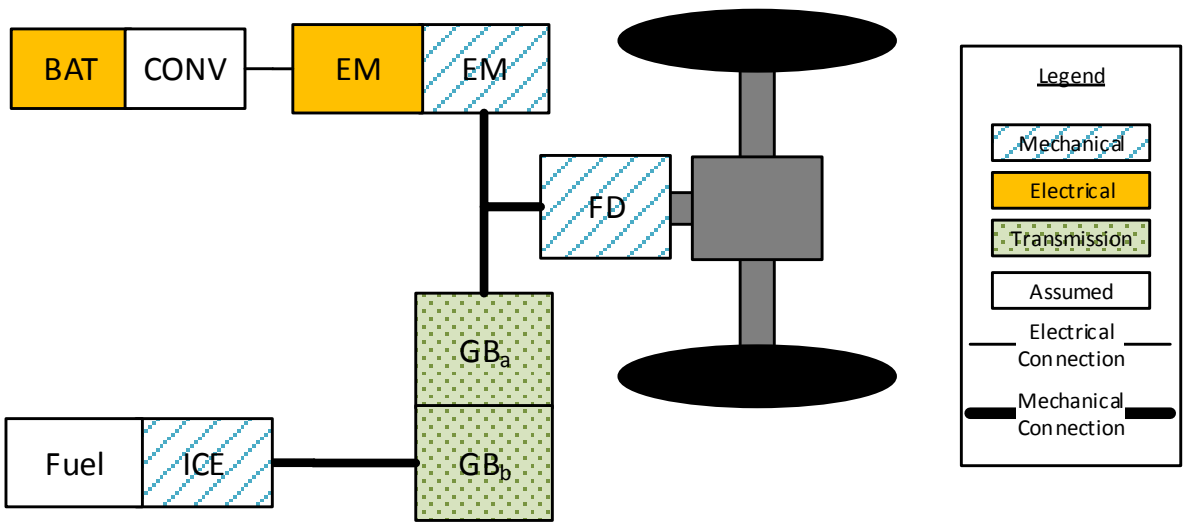

Figure 3 Parallel Hybrid Powertrain Architecture

Table 2 Model Differences between Autonomie and ASHev

\begin{tabular}{l||c||r}
\hline Feature & Autonomie (High Fidelity) & ASHev (Low Fidelity) \\
\hline Model Type & Quasi-Static, forward & Static, backward \\
E. Motor/Generator Equation & Interpolation & 4-4 Curve-fit Polynomial \\
ICE Fuel Consumption & Interpolation & Interpolation \\
PSD Efficiency & Losses (3\%) & Lossless \\
Discrete Gearbox Efficiency & Losses (interpolated) & Lossless \\
Electric Converter & Dynamic 0.95-1 Efficiency & Static 0.95 \\
Clutch & Yes & No \\
\hline
\end{tabular}

have been omitted from this paper for brevity. However, the final drive speed is exactly the same as the electric motor speed, shown in $4 \mathrm{c}$.

The inputs were the ICE torque (shown in figure 4a) and the gearbox ratio (shown in figure 4e). Shown in figure 4a, the ICE speed in the ASHev model tracks the reference speed in the Autonomie model, except when torque is reported to be negative. Shown in figure 4c the negative ICE torques represent the engine slowing down due to frictional forces. This can occur when the clutch between the engine and final drive is open, and no fuel supplied to the engine. Shown in figure 4e, Autonomie uses a gearbox ratio of ' 0 ' to represent an open clutch. ASHev uses a gear ratio of ' 1 ' instead of ' 0 ' to avoid division by zero errors. This results in a small error in the cumulative fuel consumption, which is shown in figure 4e. The fuel consumption for Autonomie and ASHev is $640.9 \mathrm{~g}$ and $637.3 \mathrm{~g}$ respectively; an acceptable $-0.55 \%$ error.

The electric motor is mechanically connected to the final drive and thus the speed tracks perfectly as seen in figure $4 \mathrm{~d}$. Shown in figure $4 \mathrm{~b}$, there is some error in the electric motor torque. The error is because the ASHev model assumes the gearbox is lossless. As the electric motor torque is equal to the difference between the final drive torque and the gearbox output torque, the small error in the gearbox output torque results in error in the electric motor torque. This torque error, and the electric motor efficiency curve fit result in a small error in the battery power output, shown in figure $4 \mathrm{f}$. 
Table 3 Parallel Model Parameters

\begin{tabular}{|l|r|}
\hline Vehicle Mass $\left(m_{v}\right)$ & $1619 \mathrm{~kg}$ \\
ICE & Prius MY04, 85kW (scaled) $1.5 \mathrm{~L}, 4$ Cylinder \\
EM & Permanent Magnet 25kW peak, 12.5kW continuous \\
Transmission & 5 Speed Manual Gearbox \\
Gearbox Ratios $\left(i_{g}\right)$ & $\{3.32,2,1.36,1.01,0.82\}$ \\
Final Drive Reduction Gear Ratio, $\left(i_{0}\right)$ & 3.93 \\
Wheel Radius & $0.287 \mathrm{~m}$ \\
Battery Type & NiMH 51x Series \\
Battery Capacity & $6.5 \mathrm{Ah}$ \\
Frontal Surface Area $\left(A_{f}\right)$ & $1.746 \mathrm{~m}^{2}$ \\
Coefficient of Drag $\left(c_{d}\right)$ & 0.3 \\
Rolling Coefficient of Friction $\left(c_{r}\right)$ & 0.015 \\
Input Variables & $\tau_{I C E}, R_{G B}$ \\
\hline
\end{tabular}

In figure $4 \mathrm{f}$, the ASHev battery power output can be seen to approximately track the reference. Regen-braking can be observed by comparing the battery output in figure 4f and electric motor speed in figure 4d; kinetic energy from the decelerating vehicle is captured, indicated by a positive battery power. Shown in figure $4 \mathrm{~h}$, the battery State-Of-Charge finishes at a higher value as expected; the lossless gearbox results in less energy spent, and a higher final SOC for the same fuel consumption. The final State-Of-Charge in the Autonomie model and ASHev is 0.643 and 0.65 , or $1.1 \%$ error.

\section{Methodology}

This section presents how each vehicle model is evaluated for fuel consumption. Combinatorics was used to generate every possible topology, then heuristics were used to quickly reduce this set to a workable number. Dynamic Programming was used to determine the optimal control law for each topology and an objective score based on fuel consumption. If there is an error when generating the model, or during Dynamic Programming, a penalty is assigned to the objective score.

\subsection{Heuristics}

Heuristics were used to eliminate invalid topologies. Each incidence matrix is formed, then checked against the following criteria:

1. By definition, an incidence matrix cannot have more than a single 1 in each column.

2. A row cannot contain a single ' 1 '. This would result in a component unconnected to the graph.

3. The first entry in the genome must be ' 1 '. This corresponds to the final drive, and reduces the number of matrix permutations.

4. It is assume that an ICE and Battery must present to be a valid HEV powertrain. 

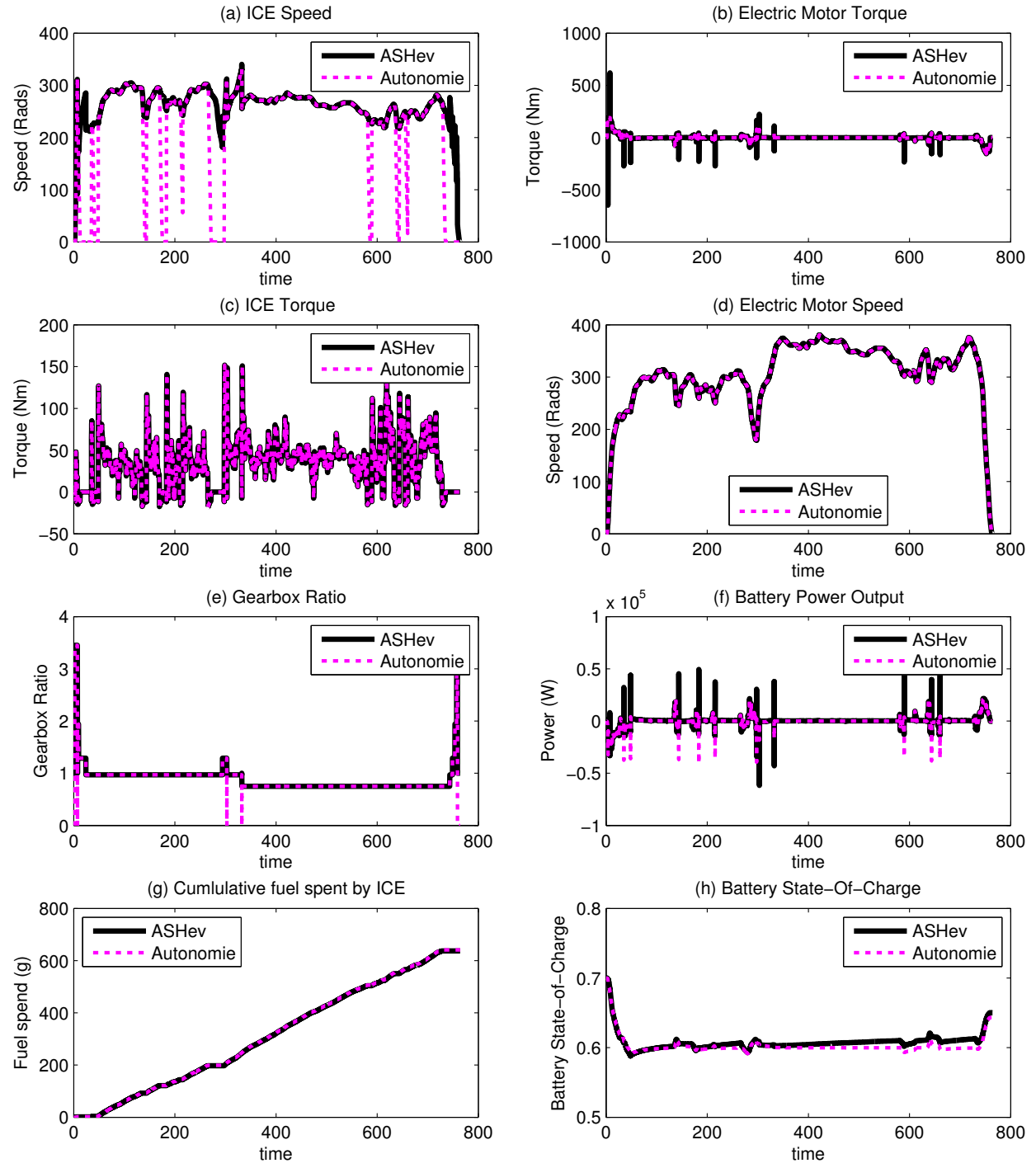

Figure 4 Parallel Hybrid Architecture Verification 
5. If the electric motor / generator is in the mechanical graph, it must also exist in the electrical graph to ensure the correct terminal equations appear in the final system of equations.

6. If included, the Powersplit Device must have connections on each gear (ring, sun, planetary carrier).

7. If included, the gearbox must have connections on both sides of the gearbox.

8. There should be no loops between the gearboxes. For example, the discrete gearbox should not connect the sun and the planetary gearset. Loops reduce the number of DOF in the system, and result in useless mechanisms.

9. The genomes that pass all heuristic criteria are then checked for isomorphs using Gauss-Jordan elimination. The resulting set should be a unique set of valid topologies.

\subsection{Dynamic Programming}

Dynamic Programming is a popular off-line method to determine the optimal control law given all future trip information (Sciarretta et al., 2004; Irani, 2009; Yuan et al., 2013). The Dynamic Program is based on Bellman's Principle of Optimality (Naidu, 2003), which states that a problem can be broken into sub-problems, and the solution to each sub-problem is dependent on the sub-problem before it. By solving backwards in time, the optimal control law for each time step is obtained. When the initial time is reached, the control law can be followed forwards in time, and the optimal control law is obtained. The implementation was based on an vectorized approach developed by Guzella (see chapter in (Lino Guzzella, 2013) for Dynamic Programming pseudocode).

The score obtained from Dynamic Programming fuel consumption over a given drivecycle, which becomes the objective score. The following are the possible outcomes and objective scores:

1. Pass is the best-case scenario, the algorithm completes without error, and the fuel consumption over a drivecycle is returned. Typical values are under 1000 .

2. FailValue is an arbitrarily set value which should be more than triple the expected fuel spent during a drivecycle.

3. Fail to find symbolic solution is the worst-case scenario, and a value based on FailValue is returned.

4. Missing entries in table occurs when a symbolic solution can be found, but the components are sized such that it cannot meet the performance constraints.

5. Pass with off target final SOC/out of bound SOC - Final SOC not on target, or SOC violates $\mathrm{SOC} \mathrm{min} / \mathrm{max}$ bounds.

\subsection{Pattern Search}

The generalized pattern search is a category of direct search methods that do not calculate or approximate derivative information to determine search directions. Pattern search was selected as the method for sizing components because it is a well known global optimization 
method with a relatively fast convergence rate (Mathworks, 2014). A non-linear method is required because the objective score can be artificially high score when a topology does not meet the performance requirements, resulting in a non-continuous problem. As the objective function is a functional (see section 4.2 for scoring method), it is not differentiable.

Pattern search systematically finds the global optimum by using exploratory moves. At each iteration, the objective function is sampled around the iterate point. The sampled point with the lowest objective score becomes the iterate point for the next iteration. Like any global optimization algorithm, convergence is guaranteed, but convergence to the global minima requires a good initial guess (Torczon, 1997; Audet and Dennis, 2003). In this work, the initial guesses for all the components are assumed to be 1 .

\section{Results and Discussion}

This section will overview the topology screening process, which is broken down into a 4 stages. The screening process is necessary because directly trying to optimize the entire design space would take an impractically long time.

The first stage removes all infeasible genomes from the gene pool by testing them against heuristics, as described in section 4.1. The second stage removes genomes that do not pass normal driving performance requirements, as tested by a partial US06 drivecycle. Three components (ICE/EM/GEN) were optimized to save computation time during the second stage. Optimization is performed to check that the topology cannot pass the constraints regardless of the component size. The third stage uses a performance drivecycle based on the PNGV (Partnership for New Generation of Vehicles) requirements to screen out poor performance topologies. For the final stage, the genomes from the third stage are ranked, and the top 20 are optimized against a combined PNGV-HWFET drivecycle. The PNGV-HWFET drivecycle ensures the vehicle can meet certain driving performance, while including average highway driving. All 5 components (ICE/EM/GEN/GB/BAT) are optimized for the final stage.

\subsection{Topology Optimization Results}

The component sizes for each topology were optimized to minimize fuel consumption for a drivecycle. 512 topologies could pass the screening heuristics in the first stage. 193 topologies passed the US06 basic driving requirements in the second stage. 159 topologies could meet the PNGV driving performance in the third stage, of which the top 20 of these topologies were selected for the final stage. For reference, common (Parallel and Prius-like Powersplit) and selected (Prius-like Powersplit with discrete gearbox before and after electric motor) topologies were included for reference. The combined PNGV-HWFET yielded different results because the drivecycle is longer and there are more opportunities to use regen-braking. The results for the optimization are sorted by fuel consumption (FC) in table 4, and diagrams of the topologies can be found in figure 5.

The optimization convergence rates varied for each topology. The topologies with fewer components took at least 12 iterations, or 75 function calls to converge. The topologies which included all the components took at max 23 iterations, and 150 function calls to converge. The optimization terminates when the step size was less than the tolerance step size of 0.1 . 
Table 4 HWFET-PNGV drivecycle results: Description of top 20 genomes seen in figure 5, where $\mathrm{FC}$ is Fuel Consumption

\begin{tabular}{|l|l|l|}
\hline Genome \# & $\begin{array}{l}\text { Component Scaling Ratios } \\
{[\text { ICE, EM, GEN, R, BAT] }}\end{array}$ & FC $(\mathrm{g})$ \\
\hline 1 & {$[0.88,0.50,0.75,0.50,0.50]$} & 614.0 \\
2 & {$[1.25,0.75,--, 0.50,0.38]$} & 617.0 \\
3 & {$[1.00,0.50,0.88,0.50,0.38]$} & 618.4 \\
4 & {$[1.25,0.50,0.38,0.50,0.38]$} & 619.0 \\
5 & {$[1.25,0.50,0.38,0.50,0.38]$} & 619.9 \\
6 & {$[1.00,0.75,0.50,0.75,0.38]$} & 620.4 \\
7 & {$[1.13,0.50,0.25,0.50,0.38]$} & 620.8 \\
8 & {$[1.25,0.50,--, 0.50,0.38]$} & 623.4 \\
9 & {$[1.25,0.88,--, 0.50,0.50]$} & 624.3 \\
10 & {$[1.00,0.50,0.50,0.50,0.63]$} & 626.5 \\
11 & {$[1.00,0.50,1.00,0.50,0.25]$} & 628.5 \\
12 & {$[1.00,0.75,--0.50,0.63]$} & 630.8 \\
13 & {$[0.88,0.50,0.63,0.50,0.38]$} & 630.9 \\
14 & {$[1.13,0.50,0.38,0.50,0.50]$} & 634.5 \\
15 & {$[1.00,1.00,0.75,0.75,0.50]$} & 635.2 \\
16 & {$[1.00,0.25,1.00,0.50,0.25]$} & 637.3 \\
17 & {$[1.13,0.50,0.25,0.75,0.50]$} & 639.1 \\
18 & {$[1.00,0.25,0.88,0.50,0.25]$} & 640.7 \\
19 & {$[1.00,0.50,0.75,0.50,0.25]$} & 641.5 \\
20 & {$[0.75,0.75,0.50,0.50,0.75]$} & 643.0 \\
21 & {$[0.88,0.75,0.50,0.50,0.50]$} & 646.9 \\
22 & {$[1.00,0.50,0.50,1.00,0.38]$} & 654.3 \\
23 & {$[1.25,1.00,1.63,--1.00]$} & 679.5 \\
24 & {$[1.00,2.00,2.00,--0.38]$} & 774.1 \\
\hline
\end{tabular}




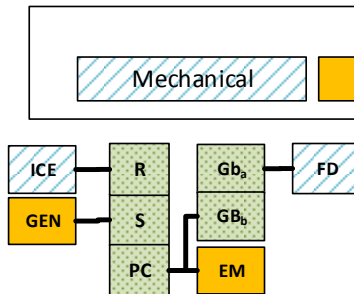

(1)

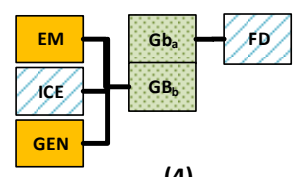

(4)

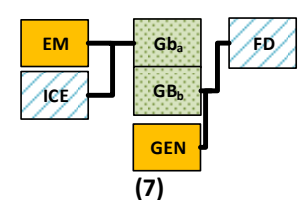

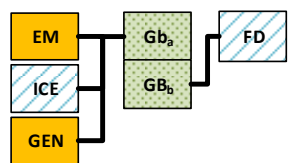

(10)

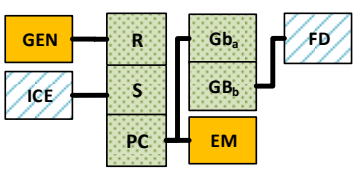

(13)

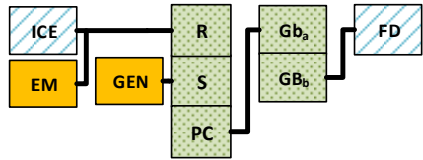

(16)

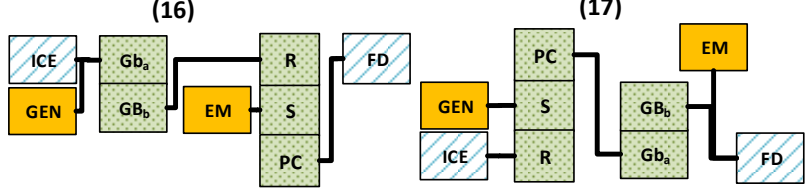

(19)

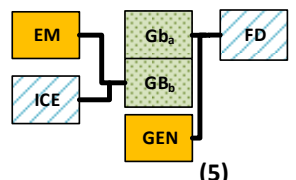

(5)

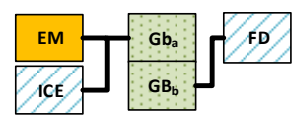

(8)

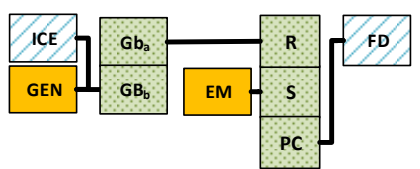

(11)

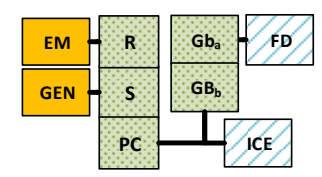

(14)
ICE

(3)

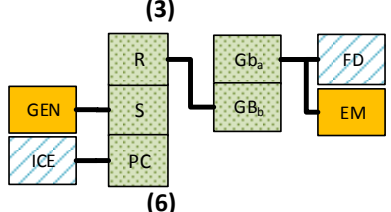

(6)

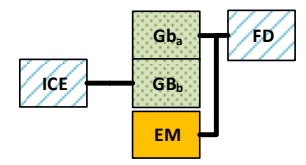

(9)

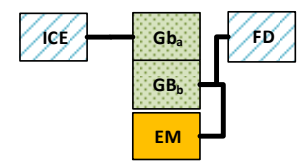

(12)

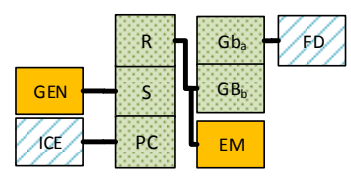

(15)

(18)

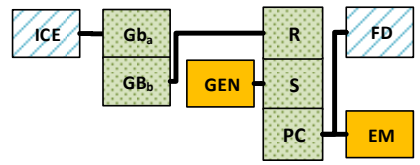

(21)
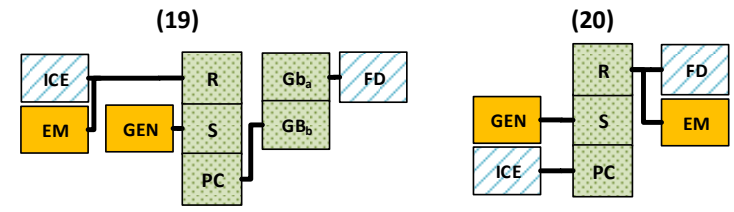

ICE GEN EM

(24)

Figure 5 Top 20 topologies obtained from the combination PNGV and HWFET drivecycle, with 4 additional topologies for reference. 


\subsection{Discussion and Interpretation of Results}

The individual topology ranking is important, but it is more important to understand which trends make these powertrains efficient. Of the top 24 evaluated topologies, there were 4 Parallel-like, 1 Series-like, 5 Complex-like, 7 Complex-Powersplit-like, and 7 Powersplitlike. Topologies \#2, 8, 9, 12 are Parallel-like, because they have an ICE mechanically connected in parallel with an electric motor and the final drive. Topology \# 24 is the reference Series hybrid powertrain and is the only Series-like topology that was evaluated. Topologies \#4, 5, 7, 10, 17 are Complex-like. Complex-like topologies are defined as 3 movers mechanically connected to a transmission (without a powersplit device). Topologies $\# 1,6,13,14,15,20,23$ are Powersplit-like, defined as one mover (ICE, EM, GEN) per gear (ring, sun, planetary carrier) on the PSD. Topologies \#3, 11, 16, 18, 19, 21, 22 are Complex-Powersplit-like, as defined by 3 movers, with more than one mover per gear on the PSD.

Long, complex geartrains suffered no penalties because mechanical losses were ignored. This skewed the results towards mechanically coupled powertrains, such as: Parallel-like, Complex-Powersplit-like and Powersplit-like topologies. The top 20 architectures contained all possible permutations of the Parallel-like topology. Complex-like and PowersplitComplex-like topologies made the majority of the genomes, however since they have more components, they more possible permutations. Electrically coupled hybrids (such as Series hybrids) performed comparatively worse because electrical inefficiencies were included. Shown in table 4, the Series Hybrid performed the worst of all 24 evaluated topologies. Powersplit-like and Complex-Powersplit-like topologies could operate as a Parallel hybrid or Series hybrid. The extra modes of operation make these topologies perform more efficiently; however, to realistically build a configuration such as \#3, additional clutches and more complex controllers would be required.

It is interesting to note that the Series (\#24) and Powersplit (\#23) powertrains did not make the top 20 topologies for the third stage. This is likely due to the objective score being a function of fuel consumption and does not include vehicle cost or controller complexity. Powertrain connectors, such as intermediate gears and driveshafts, were also not factored into this optimization. Additional constraints that could be included are: packaging, intellectual property, vehicle type, development time, safety, and chassis weight.

All of the top 20 topologies contained the discrete gearbox. Therefore, the fuel saved by adding a gearbox will always outweigh the $75 \mathrm{~kg}$ of additional mass added to the vehicle. However, the gearboxes were assumed to be lossless. In reality, they have a dynamic efficiency ranging from 95-97\%. Furthermore, extremely low efficiencies of $75-80 \%$ can be achieved if very high reduction ratios are chosen (Ehsani et al., 2010). Introducing mechanical inefficiencies into the powertrain model may yield results with fewer gearboxes.

All-electric range was not factored into this optimization, so all of the topologies have reduced their battery size to reduce vehicle weight. All-electric range could easily be incorporated in future work by changing the initial and final values of the battery SOC. Dynamic Programming always achieves the control strategy which minimizes fuel consumption while preventing the battery from exceeding the minimum and minimum and maximum SOC limits. In reality, the control strategy cannot be so ideal, and thus larger batteries are used to give flexibility for recapturing kinetic energy while braking.

Although the FD/ICE/EM/GEN could realistically be placed on the ring/sun/planet carrier gear in any combination (Ehsani et al., 2010), it was found that the planetary carrier was favored as the output gear. The Chevrolet Volt is an example of a commercially available 
HEV that uses the planetary carrier as the output gear (albeit in combination with a second planetary gear). Generally the GEN should be placed on the sun gear because it runs at the highest speed and lowest torque, and thus a smaller generator with a high speed can be used, which takes up less space and weight (Ehsani et al., 2010).

\section{Conclusions and Future Work}

A library of Hybrid Electric powertrain components was created, including: an internal combustion engine, a high torque electric motor, a low torque high speed electric motor, a planetary gearset, a discrete gearbox, and a battery. A process was developed to automatically assemble and compare the various configurations made with these components. Combinatorics was used to enumerate the different topologies, and the Graph Theoretic Method was used to assemble models. Using the steady-state models in symbolic form, Dynamic Programming was used to determine the optimal control law for each topology, thus ensuring the vehicles were compared fairly. Topologies were evaluated using a multi-stage screening process to save computation time. It was found that the parallel and Powersplit-like topologies with discrete gearboxes were most efficient. The best performing topology is a Powersplit Hybrid type, with a discrete gearbox connected to the final drive and the output gear of the planetary carrier and electric motor in parallel.

In future work, mechanical inefficiencies should be introduced. Ignoring mechanical inefficiencies may have skewed the topology to the Parallel-like rather than electrically coupled Series-like topologies.

In the future, if more components are added the number of possible topologies increase exponentially. Over a certain threshold, it may be more feasible to use the genetic algorithm to do the optimization rather than enumerate the topologies. Liu (Liu, 2004) used this approach successfully to generate planar mechanisms.

\section{Acknowledgements}

The project is funded by Toyota, Maplesoft, and NSERC. Special thanks to Dr. Joydeep Banerjee for being a great technical resource during this project.

\section{References}

Argonne National Labs. Autonomie Software R.1210, 2012.

Charles Audet and J E Dennis. Analysis of generalized pattern searches. SIAM Journal of Optimization, 13(3):889-903, 2003. URL http: / / epubs . siam.org/doi/pdf/ $10.1137 /$ S1052623400378742.

Alparslan Emrah Bayrak, Yi Ren, and Panos Y Papalambros. Design of HybridElectric Vehicle Architectures Using Auto-Generation of Feasible Driving Modes. In Proceedings of the ASME 2013 International Design Engineering Technical Conferences and Computers and Information in Engineering Conference, pages 1-9, 2013. 
C. C. Chan. The State of the Art of Electric, Hybrid, and Fuel Cell Vehicles. Proceedings of the IEEE, 95(4):704-718, April 2007. ISSN 0018-9219. doi: 10.1109/JPROC.2007. 892489. URL http: / / ieeexplore. ieee.org/lpdocs/epic03/wrapper. htm? arnumber $=4168013$.

K. Chen, A. Bouscayrol, A. Berthon, P. Delarue, D. Hissel, and R. Trigui. Global modeling of different vehicles. IEEE Vehicular Technology Magazine, 4, 2009. ISSN 1556-6072. doi: 10.1109/MVT.2009.932540.

Luigi del Re, Frank Allgower, Glielmo Luigi, Carlos Guardiola, and Ilya Kolmanovsky. Automotive Model Predictive Control. Springer, 2010. ISBN 9781849960700. doi: 10.1007/978-1-84996-071-7.

Merhrdad Ehsani, Yimin Gao, and Ali Emadi. Modern Electric, Hybrid Electric, and Fuel Cell Vehicles. CRC Press, second edi edition, 2010. ISBN 9781420053982.

Jamie Gomez, Ruben Nelson, Egwu E. Kalu, Mark H. Weatherspoon, and Jim P. Zheng. Equivalent circuit model parameters of a high-power Li-ion battery: Thermal and state of charge effects. Journal of Power Sources, 196(10):4826-4831, May 2011. ISSN 03787753. doi: 10.1016/j.jpowsour.2010.12.107. URL http: / / linkinghub . elsevier.com/retrieve/pii/s0378775311000899.

Wolfgang Novak Harald Naunheimer, Bernd Bertsche, Joachim Ryborz. Automotive Transmissions. Springer Berlin Heidelberg, 2nd edition, 1994. ISBN 9783642162138. doi: 10.1007/978-3-642-16214-5.

Theo Hofman, Soren Ebbesen, and Lino Guzzella. Topology Optimization for Hybrid Electric Vehicles With Automated Transmissions. IEEE Transactions on Vehicular Technology, 61(6):2442-2451, July 2012. ISSN 0018-9545. doi: 10.1109/ TVT.2012.2196299. URL http: / / ieeexplore. ieee.org/lpdocs/epic03/ wrapper.htm? arnumber $=6189807$.

Adam Henry Ing. Automated Topology Synthesis and Optimization of Hybrid Electric Vehicle Powertrains. Masters' thesis, University of Waterloo, 2014. URL http: / / hdl. handle. net/10012/8810.

Farzad Irani. On Dynamic Programming Technique Applied to a Parallel Hybrid Electric Vehicle. Phd thesis, Chalmers University of Technology, 2009.

V.H. Johnson. Battery performance models in ADVISOR. Journal of Power Sources, 110(2):321-329, August 2002. ISSN 03787753. doi: 10.1016/S0378-7753(02) 00194-5. URL http://linkinghub.elsevier.com/retrieve/pii/ s0378775302001945.

Akira Kawahashi and Toyota Motor Corporation. New-Generation Hybrid Electric Vehicle and Its Supporting Power Semiconductor Devices. pages 23-29, 2004.

Antonio Sciarretta Lino Guzzella. Vehicle Propulsion Systems. Springer Berlin Heidelberg, 3rd edition, 2013. ISBN 9783642359125. doi: 10.1007/978-3-642-35913-2. URL http: //link. springer.com/book/10.1007/978-3-642-35913-2/ page/1. 
Jinming Liu and Huei Peng. A systematic design approach for two planetary gear split. Vehicle System Dynamics, 48(11):1395-1412, 2010. doi: 10.1080/00423114.2010. 512634.

Yi Liu. Automated Type and Dimensional Synthesis of Planar Mechanisms Using Numerical Optimization with Genetic Algorithms. Phd thesis, University of Waterloo, 2004.

Xuerui Ma, Yong Zhang, and Chengliang Yin. Kinematic Study and Mode Analysis of a New 2-Mode Hybrid Transmission. Proceedings of the FISITA 2012 World Automotive Congress, 193, 2013. doi: 10.1007/978-3-642-33744-4. URL http: / / link. springer.com/10.1007/978-3-642-33744-4.

Mathworks. Global Optimization Toolbox, 2014. URL http://www mathworks . com/products/global-optimization/features.html.

Robert A Mcdonald. Electric Motor Modeling for Conceptual Aircraft Design. 2013.

John J. McPhee. On the use of linear graph theory in multibody system dynamics. Nonlinear Dynamics, 9:73-90, 1996. ISSN 0924-090X. doi: 10.1007/BF01833294.

John J. McPhee. Dynamics of Multibody Systems : Conventional and Graph-Theoretic Approaches, University of Waterloo. 2004. URL http: / / real. uwaterloo.ca/ $\sim$ mcphee/sd652/notes \_sd652.pdf.

John J. McPhee. Unified Modelling Theories for the Dynamics of Multidisciplinary Multibody Systems. In Jorge A.C. Ambrosio, editor, Advances in Computational Multibody Systems, pages 125-154. Springer, Lisbon, Portugal, 2 edition, 2005. ISBN 9781402033933.

John M Miller and Michael Everett. An Assessment of Ultra-capacitors as the Power Cache in Toyota THS-11, GM-Allision AHS-2 and Ford FHS Hybrid Propulsion Systems. IEEE, 2005.

Desineni Subbaram Naidu. Optimal Control Systems. CRC Press, 2003. ISBN 0849308925.

Robert L. Norton. Design of Machinery: An Introduction to the Synthesis and Analysis of Mechanisms and Machines. McGraw-Hill, New York, second edition, 1992. ISBN 0-07-237960.

Joao A. Pecas Lopes Rodrigo Garvia-Valle. Electric Vehicle Integration into Modern Power Networks. Springer Berlin Heidelberg, 2013. ISBN 9781461401339. doi: 10.1007/ 978-1-4614-0134-6.

A. Sciarretta, M. Back, and L. Guzzella. Optimal Control of Parallel Hybrid Electric Vehicles. IEEE Transactions on Control Systems Technology, 12(3):352-363, May 2004. ISSN 1063-6536. doi: 10.1109/TCST.2004.824312. URL http://ieeexplore. ieee.org/lpdocs/epic03/wrapper.htm?arnumber=1291406.

Virginia Torczon. On the Convergence of Pattern Search Algorithms. SIAM Journal of Optimization, 7(1):1-25, 1997. URL http://epubs.siam.org/doi/pdf/10. 1137 / S1052623493250780. 
Rui Wang and Srdjan M. Lukic. Dynamic programming technique in hybrid electric vehicle optimization. 2012 IEEE International Electric Vehicle Conference, pages 1-8, 2012. doi: 10.1109/IEVC.2012.6183284. URL http://ieeexplore.ieee.org/lpdocs/ epic03/wrapper.htm?arnumber $=6183284$.

Zou Yuan, Liu Teng, Sun Fengchun, and Huei Peng. Comparative Study of Dynamic Programming and Pontrayagin's Minimum Principle on Energy Management for a Parallel Hybrid Electric Vehicle. Energies, 6(4):2305-2318, April 2013. ISSN 1996-1073. doi: 10.3390/en6042305. URL http: / / www . mdpi . com/1996-1073/6/4/2305/.

Xiaowu Zhang, Chiao-ting Li, Dongsuk Kum, and Huei Peng. Prius + and Volt - : Configuration Analysis of Power-Split Hybrid Vehicles With a Single Planetary Gear. IEEE Transactions on Vehicular Technology, 61(8):3544-3552, 2012. doi: 10.1109/TVT. 2012.2208210. 